\title{
LA BANLIEUE LAUSANNOISE DÉFINIE PAR L'ÉTUDE DES MIGRATIONS ALTERNANTES DE TRAVAILLEURS
}

\author{
Jacques Barbier
}

\section{INTRODUCTION}

La banlieue, comme beaucoup des objets dont s'occupe la géographie humaine, est à peu près aussi difficile à définir qu'elle marque indiscutablement un paysage! Son nom fait toujours naître, dans l'esprit de ceux qui l'entendent ou le lisent, une série d'images parfaitement caractéristiques; mais le nombre même de ces représentations témoigne de la variété des formes qu'elle peut prendre, et de la difficulté qui s'attache à toute tentative de définition générale.

Il est sans doute une définition simple, brève et exacte:

«La banlieue, c'est la zone de contact ou de pénétration de l'habitat rural et de l'habitat urbain. (...) C'est la zone à l'intérieur de laquelle les influences urbaines s'étendent d'un mouvement centrifuge avec une force décroissante sur les campagnes». 1

Mais les qualités de concision et de simplicité se paient du prix d'une trop grande abstraction. Et une telle définition reste d'un bien faible secours à qui veut préciser les limites de la zone suburbaine.

Il faut donc renoncer à ce point de vue. La banlieue, avant tout, est un paysage, et dont on peut tenter de saisir les traits fondamentaux, les caractères spécifiques. De cette démarche sont nées plusieurs définitions. Nous n'en évoquerons ici que deux, auxquelles nous avons occasionnellement eu recours dans la suite de ce travail. Celle qui s'appuie le plus directement sur l'aspect de la banlieue est celle qui se fonde sur les modes de construction:

«Le fait fondamental (de la banlieue) c'est que les édifices contigus, qui sont la règle dans la ville, ne seront plus en banlieue que l'exception. La banlieue exige des types de construction desserrée.» 2

Une telle définition a le défaut assez grave de n'être utile que pour distinguer la banlieue de la ville, pour fixer sa limite intérieure.

Du même genre est la définition qui se fonde sur l'étude des densités. Sans doute ne s'appuie-t-elle qu'indirectement sur le paysage: la densité représente une première abstraction, une transposition de certaines particularités de ce paysage. En remarquant que «sont de la banlieue toutes les communes dont les densités cessent d'être décroissantes et qui deviennent plus importantes démographiquement par suite de l'influence de l'agglomération...»3 A. ChâtelaiN complète $P$. BONNOURE en fournissant le moyen concret de fixer une limite extérieure à la banlieue.

S'il est difficile de définir la banlieue, c'est, répétons-le, parce que la banlieue présente des aspects divers, dus à la variété de ses fonctions: Celles-ci se ramènent, en dernière analyse, à quatre.

La première a une importance aujourd'hui assez discutable: c'est la fonction de ravitaillement. En effet, si la banlieue est encore apte, dans les villes petites et moyennes, à fournir une partie importante du ravitaillement de la ville en produits maraîchers 4 , ce n'est manifestement plus le cas dans les métropoles, autour desquelles la zone de ravitaillement s'étend bien au-delà de la banlieue.

La seconde fonction de la banlieue est d'être pour le citadin une zone de villégiature. Comme la première, elle semble moins déterminante qu'autrefois. Non que le citadin ait perdu le goût de la promenade dominicale (l'exemple de Berlin-Ouest, privé de cette zone de villégiature, montre qu'elle correspond à un besoin profond) mais parce que la facilité a vec laquelle l'homme moderne se déplace a repoussé la zone de villégiature aux confins de la banlieue et dans la «zone de voisinage».

Les deux autres fonctions de la banlieue sont les plus importantes. Elles s'imposent bien plus que les premières à l'observation, parce qu'elles marquent plus profondément le paysage. Ce sont la fonction industrielle et la fonction de résidence. La «banlieue-usine» et la «banlieuedortoir».

${ }^{1}$ M. Sorre: «Les fondements de la géographie humaine.» T III, p. 319.

2 P. Bonnoure: Art. cité dans "Revue de Géographie de Lyon" 1950, fascicule 3, p. 259.

3 A. Chatelain: Art. cité dans a Revue de Géographie de Lyon»1946, fascicule 3-4, p. 118.

${ }^{4}$ C'est le cas à Lausanne. 
ports.

Or, ces deux fonctions sont liées, plus encore que les précédentes à la question des trans-

La ville qui s'accroît déborde sur la campagne. La banlieue n'est pas constituée par la région d'où la ville attire les travailleurs. Elle est la zone où refluent les personnes et les installations qui ne trouvent plus place dans la ville, gagnée de plus en plus par l'activité commerciale.

L'expansion urbaine s'accompagne d'une séparation du lieu de travail et du lieu de résidence, que ce soient les usines ou les maisons qui s'éloignent du centre. Le développement est réglé par des problèmes de circulation. Le phénomène commun à toute banlieue est celui des déplacements journaliers de travailleurs, celui des migrations alternantes. Et l'on peut dire que l'élément essentiel du paysage de banlieue est, avant les constructions desserrées ou la densité de peuplement, le tramway bondé et le flot de cyclistes des banlieues ouvrières, le garage attenant à chacune des villas d'un quartier aisé.

Notre travail repose sur l'étude des mouvements quotidiens de travailleurs. Mais il n'est pas une étude des migrations alternantes elles-mêmes, qui serait plutôt du ressort de la sociologie ou de la géographie de la circulation. Notre but est, par une localisation de ces mouvements, de déterminer les limites extérieures de la banlieue et, si possible, les limites des diverses zones (proche banlieue, grande banlieue, etc.). Il ne retient donc, des migrations, que l'aspect statistique, sans se préoccuper des moyens par lesquels elles s'effectuent, ni de la catégorie sociale des personnes qu'elles affectent.

Nous nous heurterons dans la suite de cette étude, à bien des difficultés provenant du fait que les migrations alternantes, qui sont un fait essentiel de la conurbation, ne sont qu'un phénomène isolé de son contexte. Bien souvent il faudrait recourir à d'autres critères pour définir la banlieue. Nous ne le ferons que dans les cas où cela sera véritablement indispensable.

La méthode que nous avons adoptée a ses limites et ses exigences. Parmi ces dernières, il faut citer la nécessité d'une limite interne de la banlieue: pour pouvoir faire le recensement des personnes travaillant en ville et vivant au-dehors, il faut que la ville soit définie. Or cette limite de la ville est, dans notre cas, moins évidente qu'on pourrait le penser. Nous nous sommes fondé sur les divisions administratives des communes, en admettant comme «ville» le territoire communal de Lausanne. Ceci nous a été imposé par des questions de commodité: les communes tenant, pour des raisons fiscales, un compte précis des gens travaillant sur leur territoire et domiciliés ailleurs.

Cette manière de faire ne va pas, à Lausanne, sans de très sérieux inconvénients dus à l'extension très grande de la commune qui, vers le nord, atteint les forêts du Jorat, zone qui se situe bien en-dehors de la banlieue. Il ne pouvait être question, dans un travail aussi limité, d'éviter ce handicap. Nous essaierons d'en mesurer l'importance dans un chapitre ultérieur, mais tenons, dès l'abord, à en avertir le lecteur.

\section{LES MOUVEMENTS DE TRAVAILLEURS EN DIRECTION DE LAUSANNE}

L'étude des mouvements de travailleurs n'est possible que basée sur des statistiques précises. Ces statistiques n'existent pas. Le recensement fédéral de 1950 ne donne que le nombre total des personnes travaillant à Lausanne et résidant hors de la ville (6053).

Nous avons donc dû faire une enquête personnelle, travail assez long, qui fut grandement facilité par l'obligeance avec laquelle les Autorités communales de Lausanne, Renens, Morges, et les Services cantonaux de la «Formation professionnelle» et de l'«Enseignement secondaire» mirent leurs archives à notre disposition.

La cellule administrative que nous avons choisie pour base est la Commune de Lausanne, quoique son territoire municipal dépasse largement la zone urbaine proprement dite. Nous verrons plus loin que c'est là un inconvénient mineur, les zones suburbaines du nord de la Commune n'étant pas industrialisés, et n'attirant par conséquent qu'un nombre infime de travailleurs.

Nous avons tenu compte de l'ensemble des personnes se déplaçant quotidiennement, ajoutant aux contingents de travailleurs proprement dits les apprentis et les élèves des écoles secondaires. Cela explique que le chiffre total de «migrants» auquel nos recherches ont abouti soit, avec 1140t personnes, très supérieur à celui que le recensement fédéral donne pour 1950 (6053). Les travailleurs soumis à l'impôt exerçant leur activité à Lausanne et résidant hors de la Commune, sont l'objet d'un recensement. En effet, leur impôt sur le revenu est réparti entre la Commune de Lausanne et leur commune de domicile. Cette circonstance nous a permis, en dé- 
pouillant le fichier de la "Commission d'impôt de la Commune de Lausanne» de nous fonder sur des renseignements bien plus complets que ceux qu'auraient pu fournir, par exemple, les entreprises de transport. Il n'est pas exagéré de dire que ces statistiques comprennent toutes les personnes occupées régulièrement à Lausanne, quels que soient les moyens qu'elles utilisent pour se rendre en ville.

Le fichier de la Commission d'impôt est établi d'après les listes de personnel que les entreprises (au sens le plus large du terme) fournissent au début de l'année. Ainsi, quoique nous l'ayons consulté en automne, il exprime la situation telle qu'elle était le 1er janvier 1959.

Les résultats que nous avons obtenus correspondent sans doute à la réalité pour la grande majorité des catégories de travailleurs. Toutefois, le nombre de manœuvres et d'ouvriers du bâtiment que nous avons trouvé nous paraît trop faible. Cela s'explique facilement par le fait que les ouvriers du bâtiment changent fréquemment de lieu de travail. Il est rare qu'ils passent une année entière dans la même commune. D'autre part, comme certains d'entre-eux (particulièrement les manœuvres) changent souvent d'employeur, leur recensement est spécialement difficile. La «Fédération vaudoise des Entrepreneurs», auprès de laquelle nous avons tenté de trouver des renseignements complémentaires, n'a pas pu nous les fournir. Tout en déplorant cette lacune inévitable, il convient de ne pas en exagérer les conséquences. Si l'exactitude statistique en souffre, il est certain que l'ordre de grandeur de nos résultats (qui compte plus, en définitive qu'une précision illusoire) n'est pas altéré.

Le recensement des apprentis a pu se faire avec une relative facilité. Le Service de la Formation professionnelle tient un fichier où chaque apprenti a son dossier. Nous avons eu accès à cette source de renseignements, dont l'exactitude est indiscutable, au mois de décembre 1959. Comme ce fichier est constamment tenu à jour et complété, nos statistiques reflètent la situation à cette époque. Nous n'avons toutefois pas hésité à additionner les chiffres obtenus à ceux des travailleurs et des écoliers, datant du ler janvier, parce qu'une telle manière de faire présentait l'avantage d'une représentation globale de la situation, et que les erreurs ainsi introduites semblent, ici aussi, rester négligeables: une grande part des apprentis dont nous avons eu les fiches entre les mains l'étaient déjà au début de l'année; le nombre de ceux qui commencèrent leur temps d'apprentissage au printemps doit, d'autre part, compenser dans une très large mesure les fins d'apprentissage enregistrées à la même époque. Enfin, l'on peut supposer vraisemblablement que les zones de recrutement ne varient pas beaucoup d'une année à l'autre.

Enfin en ce qui concerne les écoliers, les chiffres que nous indiquons nous ont été fournis par le Département de l'Instruction publique et des Cultes, Service de l'Enseignement secondaire, qui s'est livré, fin 1958, à une enquête sur le domicile des élèves des écoles secondaires lausannoises.

On peut se demander s'il était judicieux de tenir compte des écoliers et des apprentis dans une étude sur les migrations alternantes. On constate en effet que le rapport entre le nombre de travailleurs et le nombre d'écoliers varie beaucoup d'une commune à l'autre. Indiscutablement, les jeunes sont attirés à Lausanne pour d'autres raisons que les adultes et l'on peut remarquer que cette attraction est, en général, plus forte que celle qui s'exerce sur les travailleurs adultes. On en arrive ainsi, pour les régions éloignées, à constater un plus fort mouvement d'écoliers et d'apprentis que de travailleurs, et, particulièrement, lorsqu'une seule personne vient d'une commune éloignée, il y a de fortes chances pour qu'elle appartienne à la première catégorie.

Il n'en reste pas moins que les forts contingents d'écoliers ou d'apprentis restent l'apanage de la banlieue, dont ils soulignent le caractère. Ainsi, par exemple, 359 écoliers et 71 apprentis viennent de Pully, dont ils représentent ensemble le $3.9 \%$ de la population, et le $18 \%$ du contingent d'émigrants alternants. Une commune comme Penthalaz, sise à quelques 20 kilomètres de Lausanne, envoie 37 personnes à la ville (dont 11 apprentis et 9 écoliers), tandis qu'une commune éloignée comme Le Sentier envoie 5 écoliers, 5 apprentis et 4 travailleurs seulement à la ville.

Ces quelques exemples tirés de notre tableau général montrent comment les chiffres relatifs aux écoliers et apprentis, s'ils contribuent à atténuer l'opposition entre la grande banlieue et la zone de voisinage, ont l'avantage d'indiquer plus nettement l'extension de la zone d'influence.

Les mouvements journaliers de travailleurs se rendant à Lausanne atteignent une importance assez considérable, tant par le nombre de personnes qu'ils intéressent que par l'extension de la région qu'ils drainent.

Le nombre total des immigrants alternants est de 11404, dont 8977 travailleurs, 900 apprentis et 1527 écoliers. Remarquons que, par rapport aux chiffres du recensement fédéral de 1950, le nombre de travailleurs participant aux mouvements pendulaires a augmenté de 2924 unités, soit de $48 \%$, tandis que la population de la Commune de Lausanne passait de 106807 à 121210 (augmentation de 13,5\% seulement), ce qui est parfaitement conforme à la loi de l'accroissement des villes par la périphérie. 


\section{Liste des communes dont plus de 50 habitants sont occupées à Lausanne}

\begin{tabular}{|c|c|c|c|c|c|c|c|c|}
\hline & Population & & leurs & & ntis & Ecoliers & Total & $\%$ \\
\hline & & $\mathrm{H}$ & $\mathbf{F}$ & $\mathrm{H}$ & $\mathrm{F}$ & & & \\
\hline Belmont & 508 & 55 & 16 & 4 & 2 & 13 & 90 & 17.00 \\
\hline Bussigny & 2138 & 114 & 40 & 8 & 11 & 22 & 195 & 9.10 \\
\hline Chavannes & 1559 & 104 & 44 & 9 & 10 & 16 & 183 & 12.00 \\
\hline Chexbres + Puidoux & 2791 & 54 & 21 & 5 & 3 & 6 & 89 & 3.00 \\
\hline Cossonay & 1251 & 18 & 16 & 7 & 4 & 16 & 61 & 5.00 \\
\hline Crissier & 2834 & 169 & 55 & 10 & 6 & 19 & 259 & 9.10 \\
\hline Cully & 1309 & 51 & 29 & 7 & 3 & 30 & 120 & 9.20 \\
\hline Echallens & 1379 & 35 & 17 & 3 & 9 & 15 & 79 & 3.60 \\
\hline Ecublens & 2122 & 139 & 55 & 8 & 5 & 10 & 217 & 10.00 \\
\hline Epalinges & 1213 & 125 & 40 & 7 & 2 & 20 & 194 & 16.00 \\
\hline Grandvaux (Genève) & 870 & 62 & 22 & 3 & 2 & 27 & 116 & 13.00 \\
\hline Lonay & 945 & 33 & 19 & 3 & 5 & 6 & 66 & 6.90 \\
\hline Lutry + La Conversion & 3376 & 183 & 78 & 14 & 12 & 50 & 462 & 16.70 \\
\hline Le Mont síLausanne & 1581 & 160 & 35 & 9 & 6 & 21 & 231 & 14.60 \\
\hline Montreux & & 83 & 38 & 6 & 1 & 37 & 165 & \\
\hline Clarens & & 50 & 22 & 3 & 4 & 14 & 93 & \\
\hline Territet & & 13 & 5 & & & 5 & 23 & \\
\hline Veytaux & & 6 & 2 & & & 1 & 9 & \\
\hline Montreux agglomération & 17844 & & & & & & 290 & 1.70 \\
\hline Morges & 8011 & 208 & 104 & 23 & 21 & 59 & 415 & 5.20 \\
\hline Moudon & 2701 & 36 & 12 & 5 & 3 & 8 & 64 & 2.50 \\
\hline Nyon & 6792 & 32 & 17 & 2 & 6 & 22 & 79 & 1.10 \\
\hline Orbe & 3424 & 26 & 9 & 8 & 2 & 14 & 59 & 1.60 \\
\hline Oron & 882 & 30 & 9 & 3 & 1 & 14 & 57 & 6.30 \\
\hline Paudex & 718 & 62 & 27 & 1 & & 7 & 97 & 13.00 \\
\hline Prilly & 7064 & 848 & 370 & 42 & 38 & 140 & 1438 & 20.00 \\
\hline Pully & 11333 & 1491 & 467 & 42 & 29 & 359 & 2388 & 21.00 \\
\hline Renens & 9681 & 755 & 350 & 53 & 37 & 141 & 1336 & 13.80 \\
\hline Rolle & 2821 & 36 & 4 & 3 & 3 & 5 & 51 & 1.80 \\
\hline Saint-Prex & 1782 & 28 & 11 & 5 & 3 & 9 & 56 & 3.10 \\
\hline Saint-Sulpice & 1075 & 124 & 22 & 6 & 3 & 34 & 189 & 17.50 \\
\hline La Tour-de-Peilz & 6434 & 70 & 32 & 6 & 2 & 26 & 136 & 2.10 \\
\hline Vevey + Corseaux & 16980 & 243 & 85 & 25 & 7 & 81 & 441 & 2.60 \\
\hline Yverdon & 15634 & 95 & 43 & 17 & 9 & 8 & 172 & 1.10 \\
\hline
\end{tabular}

La répartition géographique de ces 11404 personnes apparaît sur notre carte no 1 . Autour de la ville se trouve une zone où le nombre d'émigrants par commune décroît concentriquement. Ensuite les forts contingents se groupent exclusivement le long des bonnes voies de communication. Les localités situées à l'écart de ces voies se signalent par un nombre d'émigrants alternants très faible; il est remarquable toutefois que les régions qui ne fournissent aucun travailleur à Lausanne soient assez rares.

Au tableau général sont indiquées, par ordre alphabétique, toutes les communes du Canton de Vaud envoyant des travailleurs à Lausanne. Les cantons voisins n'envoient que de faibles contingents, que nous avons négligés, à l'exception de Genève (56 personnes). Il nous a toutefois paru utile de dresser ici une liste, par ordre d'importance du contingent d'émigrants alternants, des communes qui dépassent 100 :

$\begin{array}{lrll}\text { Pully } & 2388 & \text { Ecublens } & 217 \\ \text { Prilly } & 1438 & \text { Bussigny } & 195 \\ \text { Renens } & 1336 & \text { Epalinges } & 194 \\ \text { Lutry } & 465 & \text { St-Sulpice } & 189 \\ \text { Vevey + Corseaux } & 441 & \text { Chavannes } & 183 \\ \text { Morges } & 415 & \text { Yverdon } & 172 \\ \text { Montreux (agglomération) } & 290 & \text { La Tour-de-Peilz } & 136 \\ \text { Crissier } & 259 & \text { Cully } & 120 \\ \text { Le Mont } & 231 & \text { Grandvaux } & 116\end{array}$

Cette liste contient, à côté des communes qui font indiscutablement partie de la banlieue, toutes les villes les plus importantes du Canton, quoique leurs contingents 


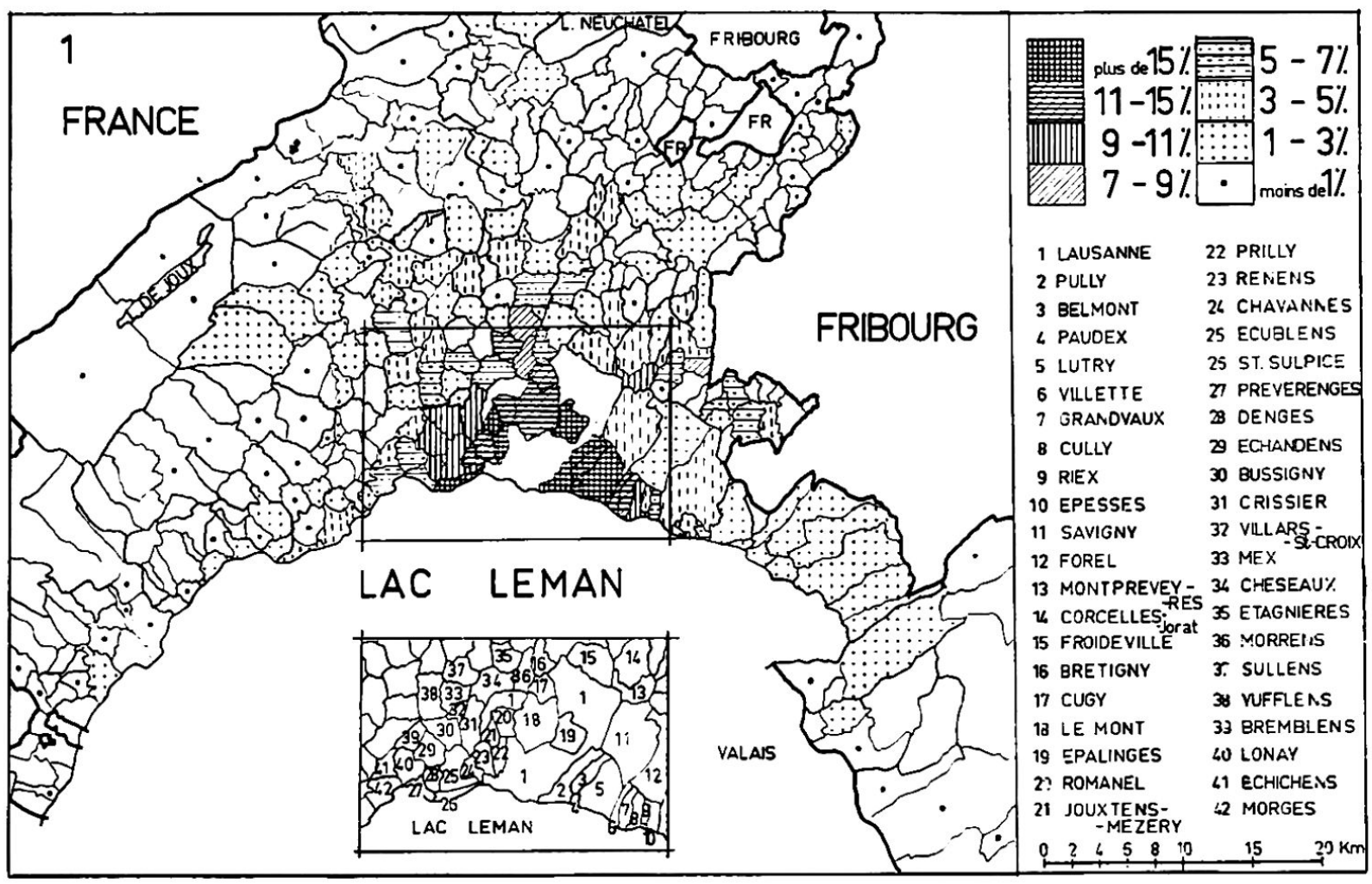

1. Provenance, en $\%$ de leur population de résidence, des personnes occupées à Lausanne.

n'aient, en regard de leurs chiffres de population, qu'une faible importance. C'est pour éviter des disproportions semblables que nous avons calculé les proportions (en \%) des émigrants alternants par rapport aux populations totales des communes. C'est l'étude de ces indices relatifs qui sera le sujet de notre prochain chapitre.

\section{LA RÉPARTITION, PAR COMMUNES DE DOMICILE,} DES TRAVAILLEURS LAUSANNOIS, ET LA LIMITE DE LA BANLIEUE

La représentation cartographique: Le travail de statistique terminé, il a été facile de représenter cartographiquement les résultats. La seule question qui s'est posée réellement fut de savoir s'il fallait tenir compte des chiffres absolus de «migrants», ou les exprimer en fractions de la population totale des communes.

Les deux manières de faire présentent des inconvénients. Ni l'une ni l'autre ne donne une image exacte de la réalité.

L'expression du résultat en \% de la population totale a l'avantage de mesurer, en quelque sorte, l'intensité de l'influence urbaine. Une rue où le $20 \%$ de la population est occupée en ville présente un tout autre aspect que celle où seulement $3 \%$ des habitants remplissent la même condition. Cependant, la représentation proportionnel̀le a le défaut assez grave de mettre sur le même pied la véritable agglomération suburbaine, dont la densité de population est élevée, et le village, planté au milieu de son terroir presque intact, auquel se sont ajoutées, attirées par le calme ou le panorama, quelques villas dont l'importance équilibre très vite, sur nos statistiques, un village entier. Nous en avons un cas concret. Sur la carte que nous avons dessinée, le Mont et Renens ont la même teinte, quoiqu'il s'agisse de deux communes extrêmement différentes, la première ayant une densité de 1,6 habitants par ha. et la deuxième de 34,3 habitants par ha.

La seconde solution, celle de la représentation des nombres absolus d'émigrants alternants évite ces erreurs. Mais elle présente des défauts qui la rendent presque inutilisable. Que signifie en effet le nombre des personnes employées en ville si l'on ne connaît 
la population totale? Comment différencier Belmont, avec 90 «migrants», d'Echallens (79) ou de Paudex (97)? Quelle valeur attribuer aux 441 de Vevey et aux 337 de Lutry? La représentation en chiffres absolus donne une carte qui n'est que d'une faible lisibilité et qui peut induire en erreur. C'est pourquoi nous avons préféré, en définitive, calculer la proportion que représentent, dans chaque commune, les habitants travaillant à Lausanne.

La limite statistique de la banlieue; première approximation: La carte (no 1), qui concrétise le résultat de nos enquêtes, ne fait que poser clairement les données du problème, sans le résoudre. Pour cela, il faut l'interpréter, et c'est ici que commencent les vraies difficultés. Toutes les localités qui envoient quotidiennement des travailleurs à Lausanne ne font pas partie de sa banlieue. «Les migrations alternantes ne deviennent phénomène de banlieue qu'à partir d'un certain taux».5 Mais à partir de quel taux?

Deux chiffres ont, à notre connaissance, été proposés pour l'interprétation des mouvements de travailleurs. M. R. Clozier estime que les communes qui (entre autres conditions) comptent plus du $10 \%$ de leur habitants occupés en ville font «indiscutablement» partie de la banlieue ${ }^{6}$. M. MAX SORRE, d'autre part, pense que la zone d'influence urbaine s'étend aux localités dont plus de $10 \%$ des travailleurs exercent leur activité en ville. ${ }^{7}$

Il est bien évident que, de ces deux taux, celui qui nous intéresse surtout est celui qui définit la banlieue, le premier. Toutefois, afin d'exploiter au maximum le résultat de nos statistiques, nous nous occuperons dans un chapitre très bref, de la zone d'influence, que nous essayerons de limiter par l'étude des mouvements de travailleurs.

Contentons-nous pour le moment, du critère que propose M. R. Clozier. Il et nécessaire de rappeler que celui-ci ne présente pas ce taux de $10 \%$ comme une loi rigoureuse et généralement valable, mais comme une constatation faite à l'issue d'une étude régionale de la banlieue parisienne. Si nous appliquons, comme nous le ferons, ce critère à la banlieue lausannoise, ce n'est qu'à titre provisioire, nous réservant de l'adapter pour lui faire mieux rendre compte de la réalité.

Si l'on dresse la liste des localités dont le «taux» est supérieur à $10 \%$, on obtient: Pully (21\%); Prilly (20\%); Jouxtens-Mézery (17,6\%); St-Sulpice (17,5\%); Belmont (17\%); Lutry (16,7\%); Epalinges (16\%); Le Mont (14,6\%); Romanel $(14 \%)$; Renens (13,8\%); Paudex, Cugy et Grandvaux (13\%); Chavannes $(12 \%)$; Villette et Etagnières ( $11 \%$ ) ; enfin Ecublens (10\%). Cette liste paraît bien, à première vue, comprendre les communes de la banlieue. Mais quiconque connaît la région lausannoise y distingue toute une série d'anomalies.

Les faibles taux de l'agglomération de Renens-Gare: La plus importante est sans doute l'effacement très net de la banlieue ouest de la ville, au profit de banlieues résidentielles éloignées, comme Belmont, Grandvaux, Cugy, etc. Cette anomalie n'est pas extraordinaire, mais inhérente à la méthode que nous avons employée pour définir la banlieue.

En effet, la zone ouest de la banlieue est une région nettement industrielle. Elle offre par conséquent à sa population des possibilités de travail sur place, qui réduisent le nombre des départs quotidiens pour la ville. L'influence des industries est encore accentuée par le fait que leur présence, en rendant une région moins agréable, s'accompagne d'une baisse générale des loyers. Ainsi un ouvrier d'usine travaillant à Renens trouve à Renens les loyers les moins chers de toute l'agglomération lausannoise.

Les zones purement résidentielles (par exemple celles de la banlieue Est, d'où topographie plus difficile et l'opposition des habitants exclut l'industrie) ${ }^{8}$, se signalent par

5 R. Clozier: Ouvrage cité, p. 259.

6 R. Clozier: Ouvrage cité, p. 259.

7 M. Sorre: Ouvrage cité, p. 369.

$8 \mathrm{La}$ Commune de Paudex fait exception. Elle est la seule. 
des coefficients très hauts. Les chiffres du recensement fédéral de 1950 nous apprennent ainsi que pour 1000 habitants de la commune exerçant une profession, on comptait 441 émigrants alternants et 72 immigrants alternants à Pully, tandis qu'à Renens ces chiffres étaient respectivement 372 et 434 ! 9

Il est impossible de ne pas tenir compte de cette remarque, et il faut corriger en conséquence les indications de notre carte; cela nous amène à faire entrer immédiatement Crissier $(9,1 \%)$ dans la liste des communes de banlieue. Enfin cette remarque nous incitera à la prudence en ce qui concerne la banlieue ouest, où il serait dangereux de se fier aux chiffres seuls.

Trois cas discutables: La liste que nous avons établie contient d'autres cas douteux: ceux des communes qui, bien que plus du $10 \%$ de leurs habitants travaillent à Lausanne, ont une population et une densité si faible qu'il semble surprenant de les voir comptées dans la banlieue. L'une des caractéristiques de la zone suburbaine semble, en effet, être la rapide augmentation de population, accompagnée d'une dégradation assez marquée du territoire rural. Or, dans notre étude, nous avons affaire à des communes encore presque entièrement rurales, où les éléments urbains sont limités à quelques maisons du village.

Les cas les plus frappants sont ceux d'Etagnières, de Villette et de Jouxtens-Mézery, que nous étudions ici plus en détail.

Etagnières fait nettement tache, avec ses $11 \%$ de travailleurs urbains dans une région où les taux sont bien plus bas. La commune est séparée du groupe des localités suburbaines par Cheseaux $(6,5 \%)$ et Morrens (7,5\%). La première hypothèse qui explique cette bizarrerie est celle qui voudrait que ce village bénéficie d'une situation privilégiée au point de vue des moyens de transports. Il n'en est rien. S'il est vrai que le village d'Etagnières a sur Morrens l'avantage considérable d'être sis le long de la voie ferrée, c'est un avantage qu'il partage avec Cheseaux et Assens, sans que ces deux voisins n'aient des taux aussi élevés. Le caractère exceptionnel subsiste donc.

L'aspect du village ne diffère pas beaucoup, du reste, de celui de Cheseaux ou d'Assens. La grande majorité des maisons est de type rural. Il n'y a pas d'industrie. Il n'y a pas d'activité maraîchère due à la proximité de la ville (voir carte no IV). Seules quelques constructions récentes, par leur aspect moderne, se distinguent nettement du reste, le long de la route cantonale, preuve difficile à nier d'un peuplement suburbain.

Il nous paraît toutefois difficile de compter dans la banlieue une localité qui n'envoie à la ville que 26 personnes pour une superficie communale de 374 ha., entièrement livrée à l'agriculture (densité 0,6 ). Enfin, et cette dernière observation est peut-être la plus décisive, le village compte, en 1958, 16 habitants de moins qu'en 1910 et 30 de moins qu'en 1860. De toutes les communes que contient cette première liste, elle est la seule (avec Villette) qui perde encore des habitants entre 1950 et 1958 . Or «sont de la banlieue toutes les communes dont les densités cessent d'être décroissantes et qui deviennent plus importantes démographiquement par suite de l'influence de l'agglomération». ${ }^{10} \mathrm{Ce}$ 'est manifestement pas le cas, et il ne faut pas encore compter Etagnières dans la banlieue, malgré ses $11 \%$, et quoique des indices nombreux laissent penser qu'elle en fera bientôt partie.

Villette ne semble pas, au premier abord, se trouver dans une situation très différente. La population n'est que de 361 habitants, le nombre des travailleurs occupés à Lausanne de 42. La commune a perdu 16 habitants entre 1950 et 1958, mais la population actuelle est supérieure à celle de 1910 (339 habitants). La surface communale

${ }^{9}$ Notre enquête, effectuée sur les statistiques du 1er janvier 1959, montre qu'à Renens l'équilibre s'est modifié. En 1959, nous comptons en effet 1336 habitants de"Renens occupés à Lausanne, alors que la localité de banlieue n'attire que 1266 travailleurs. La différence avec Pully reste frappante.

10 A. Chatelain. Dans * Revue de Géographie de Lyon*. 1946, fasc. 3-4, p. 118. 
n'est que de 131 ha., la densité atteint presque 2,8 par ha. La localité est entourée de communes au taux plus élevé, qui font indiscutablement partie de la banlieue.

Enfin les habitations de caractère suburbain, les villas, sont nombreuses et groupées (le long de la route Lausanne-Vevey, entre la chaussée et la rive du lac). Le reste de la commune est occupé par les vignes (sur tout le coteau) et par des champs (situés sur le plateau, très loin du chemin de fer). Or, il est certain que les régions viticoles offrent une résistance exceptionnellement forte à l'expansion urbaine. Nous sommes donc porté à croire que la régression que marque le chiffre de la population ces dernières années est un phénomène passager, touchant plus le village vigneron que la zone des constructions suburbaines. Il serait donc faux d'exclure cette commune de la banlieue.

Nous touchons ici à une difficulté dont il faut dire quelques mots tout de suite. L'étude des mouvements de travailleurs étant basée sur les limites administratives amène à considérer les communes comme des entités, ce qui ne va pas sans inconvénients. Ainsi, lorsque nous disons que Pully fait partie de la banlieue immédiate de Lausanne, cette affirmation n'est valable que pour une partie de la commune, la partie habitée, et ne s'applique pas aux régions, parfois très proches, qui sont, dans le haut vallon de la Paudèze, par exemple, des solitudes forestières assez inhospitalières et offrant un dépaysement complet. Nous continuerons cependant à parler des communes comme d'unités, nous réservant de distinguer, dans un chapitre ultérieur ce qui, à l'intérieur des communes que nous aurons classées dans la banlieue, est vraiment touché par l'expansion urbaine de ce qui y échappe.

Un troisième cas douteux existe: celui de Jouxtens-Mézery. Là aussi la population est faible (268 habitants) et la densité médiocre pour une zone suburbaine, n'atteint pas 150 habitants au $\mathrm{km}^{2}$. Le nombre actuel des habitants est inférieur à ce qu'il était en 1910, mais on note une augmentation pendant les dix dernières années (1950: 245 habitants). Cette augmentation est d'autant plus remarquable que, comme l'écrivait C. BIERMANN en 1952: «Les grandes propriétés d'étrangers comme de riches vaudois accaparent tant de place dans ces deux hameaux et entre eux que l'expansion urbaine y est quasi impossible».11

Nous rencontrons ici un autre type de banlieue: celle qui, réservée à la résidence de luxe, est par là même destinée à garder une faible densité de population et une apparence de parc.

Nous n'avons étudié ici dans le détail que les cas les plus discutables. Il ne nous semble pas nécessaire de continuer l'examen individuel de toutes les communes que nous avons citées, estimant que, pour les autres, les rézultats statistiques sont assez nets pour qu'on puisse faire l'économie de telles discussions.

La vraie difficulté: la limite ouest. Il est admis jusqu'ici que nous appliquons en principe la règle proposée par M. R. Clozier: les communes dont plus de $10 \%$ des habitants travaillent en ville sont de la banlieue, les autres n'en sont pas. Nous avons discuté ensuite quelques cas particuliers qui nous paraissaient le mériter: c'est ainsi que nous avons décidé d'augmenter notre liste de Crissier, et de l'alléger d'Etagnières, où les statistiques semblaient en contradiction avec la réalité. Il va s'agir maintenant de faire la critique d'ensemble de la banlieue ainsi définie.

L'agglomération constituée par ces communes est assez imposante: de Grandvaux, à la Venoge elle s'étire sur une quinzaine de kilomètres, tandis que sa plus grande largeur atteint, entre Ouchy et Cugy, 8 kilomètres. Elle forme un tout compact, aux formes pleines, ce qui provient, répétons-le, du fait que nous considérons la commune comme une entité: la zone de banlieue proprement dite est réduite, en périphérie, à d'étroites bandes collées aux axes de communications.

A ce point de notre étude, ce qui nous intéresse avant tout est de savoir si la limite choisie $(10 \%)$ correspond à quelque chose de concret. L'étude de notre carte no 1 ne permet pas de donner une réponse unique. D'une région à l'autre, les conditions varient.

A l'est de la ville, le critère choisi paraît tout à fait satisfaisant. On remarque une brusque chute du nombre des travailleurs soumis aux mouvements migratoires pendu-

11 C. Biermann: «Le Canton de Vaud». Lausanne 1952, p. 196. 
laires. Pully, Paudex, Belmont, Lutry, Villette et Grandvaux se tiennent au-dessus de $10 \%$ (en réalité au-dessus de $11 \%$ ). Les communes qui leur sont adjacentes à l'extérieur de la limite ont des taux très différents: Savigny (3\%), Forel $(1,2 \%)$, Puidoux-Chexbres (3\%), Riex (3,6\%), Epesses (6\%). Un seul cas plus délicat: celui de Cully avec $9,2 \%$. Faut-il l'exclure du groupe des plus de $10 \%$, ou faut-il l'y joindre? Nous penchons pour la deuxième solution, remarquant qu'il en est plus proche que des $6 \%$ d'Epesses. Mais laissons pour le moment la question en suspens.

Au nord de la ville, la situation est beaucoup moins schématique. Une première difficulté, et non des moindres, provient du fait que le territoire communal lausannois dépasse largement la ville et sa banlieue vers le nord. L'étude que nous avons faite, basée sur les divisions administratives, perd toute efficacité. Nous devrons, dans un chapitre nouveau, tâcher de fixer les limites, dans cette région, à l'aide d'autres moyens.

Là où les communes suburbaines donnent de nouveau prise à nos statistiques (Le Mont, Romanel, Cugy), nous apercevons une limite qui, de nouveau, est assez nette. Le groupe de communes à taux élevé d'émigrants alternants s'oppose à un groupe à taux bien différent Jouxtens-Mézery (17,6\%), Romanel (14\%), Le Mont $(14,6 \%)$ et Cugy (13\%) se distinguent nettement de Cheseaux (6,5\%), Morrens $(7,5 \%)$ et Bretigny $(6 \%)$. La limite idéale de $10 \%$, ici aussi, correspond à une chute réelle.

Il n'en est pas de même à l'ouest de la ville. Ici la césure s'atténue. La séparation à $10 \%$ ne s'impose plus du tout, au contraire. Nous arrivons dans une zone où se fait sentir de façon perturbatrice l'influence d'une autre ville, Morges. Nous verrons ailleurs comment s'exerce cette influence.

Si l'on en revient à la limite que, en première approximation, nous avons fixée à la banlieue, force nous est de reconnaître qu'elle est ici absolument arbitraire. Crissier ( 9,1 mais industrielle), et Ecublens ( $10 \%$ mais médiocrement industrialisé) seraient de la banlieue, et Bussigny ( $9,1 \%$ faible industrie), Echandens ( $9 \%$ ) et Denges $(9,3 \%)$ n'en seraient pas? Cela semble difficile à admettre. Il faut donc modifier le critère que nous avions admis au début de ce chapitre.

La même région nous permet de trouver une solution. Il n'existe pas de césure entre les communes dont le taux est supérieur ou inférieur à 10\%. Mais il en existe une autre à $9 \%$.

Si l'on fait, dans cette région, la moyenne des taux des communes situées de part et d'autres de la ligne idéale de $10 \%$, on obtient $11,2 \%$ pour les communes situées à l'intérieur (Renens-Chavannes-Ecublens-Crissier) et $9,125 \%$ pour celles qui sont situées à l'extérieur (Denges-Echandens-Bussigny). Si l'on fait la moyenne des communes situées à l'extérieur de la ligne de $9 \%$, on obtient 5,6\% (Préverenges-Lonay-Bremblens-Vufflens-la-Ville). Nous omettons à dessein Villars-Ste-Croix, Aclens et Romanel sur Morges qui feraient baisser cette moyenne, mais ne sont adjacentes que sur de très petites sections. Ce résultat arithmétique semble assez probant et nous pousse à écrire que, dans le cas de l'agglomération lausannoise, la banlieue est formée de toutes les communes dont plus du $9 \%$ des habitants exercent leur activité à Lausanne.

Ce léger abaissement du taux que nous avions admis n'entraîne, dans les autres secteurs, que de faibles modifications: il augmente la banlieue de Cully et de Montpreveyres. En ce qui concerne Cully, le cas ne soulève pas d'objections: nous avons vu plus haut qu'il faisait déjà indiscutablement partie du groupe des communes s'opposant, par le nombre de leurs habitants travaillant à Lausanne, aux communes rurales.

L'inclusion de Montpreveyres dans la banlieue nous semble, à première vue, plus hasardeuse. Mais l'absence générale de renseignements dans la région nord, due à l'extension de la Commune de Lausanne nous empêche de décider immédiatement. Nous le ferons lorsque nous tenterons, dans un autre chapitre, de fixer les limites de la ville vers le nord. 
Nous sommes parvenu, au cours de ce chapitre, à fixer une limite à la banlieue lausannoise, limite encore bien imprécise dans la région ouest, et qu'il faut consolider par d'autres arguments. On pourrait en effet objecter que Renens, ville industrielle de 10000 habitants est également un centre d'attraction de travailleurs, et que son attraction pourrait bien bouleverser notre limite. C'est pour répondre à cette objection que nous étudierons, dans le prochain chapitre, les mouvements pendulaires dirigés vers Renens.

Quant à la ville de Morges, elle pose un problème semblable, par l'existence d'une banlieue qui, dans son extension, vient limiter celle de Lausanne. Où placer la limite entre les deux influences? Il est impossible de le dire sans connaître l'étendue de la banlieue morgienne vers l'Est et l'importance des échanges de travailleurs existant entre Lausanne et Morges. Cette étude fera l'objet de notre quatrième chapitre.

\section{RENENS: UN CENTRE SECONDAIRE D'ATTRACTION DES TRAVAILLEURS}

Les mouvements de travailleurs dirigés vers la ville ne sont pas la seule forme que puissent revêtir les déplacements de main-d'œuvre. Ils ne constituent que ce qu'on appelle le «courant normal», auquel s'opposent le «courant inverse» (celui des personnes qui habitent en ville et travaillent dans la banlieue) et le «courant transverse ou inter-banlieue» (mouvements entre deux communes de la banlieue). Ces deux derniers courants sont, en général, moins importants que le courant normal. ${ }^{12}$

Lausanne n'est ni une grande ville, ni une ville industrielle. Son expansion n'est pas suffisamment rapide pour s'imposer à tous les secteurs périphériques. Les conditions locales vont donc jouer un rôle primordial dans son développement. C'est ainsi que la banlieue Est doit sa croissance aux agréments du site avant tout. Inversément, l'industrie est attirée par les reliefs plus faciles de la région ouest. Cette attraction, renforcée par celle qu'exerce la présence de la gare de triage explique que la région de Renens soit la seule concentration industrielle importante de la zone suburbaine, ${ }^{13}$ et par conséquent, la seule agglomération susceptible d'employer un nombre important de travailleurs étrangers.

L'agglomération industrielle de la banlieue ouest s'étend sur les territoires de quatre communes: Renens, Crissier, Chavannes et Ecublens. Nous avons été contraint par le manque de temps de limiter notre enquête à la première nommée, la plus importante de beaucoup, celle qui a donné son nom à l'ensemble et qui compte deux fois plus d'habitants que les autres réunies (si l'on ne tient compte que des parties vraiment industrielles de Crissier et d'Ecublens). Nous avons également laissé de côté les mouvements d'apprentis et d'écoliers ${ }^{14}$, limitant notre étude aux seuls travailleurs adultes. Nos statistiques se fondent sur les listes d'effectifs fournies, au début de 1959, par les entreprises de la commune.

Les résultats sont numériquement importants: la commune occupe sur son territoire 1265 travailleurs «étrangers» (Rappelons que le nombre des habitants de Renens travaillant à Lausanne est de 1336). Comparé à la population de la commune, ce contingent d'immigrants alternants, qui en représente le $12 \%$, est le plus important que celui qu'attire Lausanne ( $5 \%$ seulement de la population de la capitale).

12 Cette terminologie est empruntée à l'article de R. Clozier: « Essai sur la banlieue», paru dans * La Pensée», 1945.

18 Il est vrai que Paudex présente aussi un paysage industriel assez caractérisé. Mais il ne s'agit que d'une concentration minuscule de petites entreprises dont l'influence sur l'ensemble des mouvements de travailleurs est très faible.

14 Ces derniers sont inexistants, car il n'y a pas d'école secondaire à Renens. 
II. Liste des communes dont plus de Io habitants sont occupés à Renens

\begin{tabular}{lrrrr} 
& Population & \multicolumn{2}{c}{ Travailleurs } & $\%$ \\
Bussigny & & $\mathrm{H}$ & $\mathrm{F}$ & \\
Chavannes & 2138 & 32 & 6 & 1.77 \\
Crissier & 1559 & 99 & 10 & 6.99 \\
Ecublens & 2834 & 136 & 16 & 5.37 \\
Lausanne & 2122 & 81 & 4 & 4.01 \\
Morges & 121210 & 534 & 64 & \\
Prilly & 8011 & 25 & 4 & \\
Pully & 7064 & 63 & 6 & 1.00 \\
Saint-Sulpice & 11333 & 24 & 2 & \\
Vevey & 1075 & 14 & & 1.30 \\
& 15714 & 16 & &
\end{tabular}

Des 1265 travailleurs qui, tous les matins, se rendent à Renens, 598 habitent Lausanne, portant l'importance du courant inverse, dans ce cas précis (et unique) à près de $50 \%$ du mouvement normal.

Le reste se répartit dans les communes environnantes; Crissier (152), Chavannes (109), Ecublens (85), Prilly (69) fournissent nettement les plus gros contingents. Ensuite viennent Bussigny (38), Morges (29), Pully (26), etc. (tableau complet à la page VII). Si l'on exprime le nombre d'émigrants alternants en \% de leur population, les communes se classent dans l'ordre suivant, qui met nettement en évidence les autres communes de l'agglomération:

\section{Chavannes $7 \% \quad$ Crissier $5,37 \%$ Ecublens $4 \%$}

Bussigny compte moins de $2 \%$, Prilly, qui est avant tout tourné vers Lausanne, $1 \%$. Morges est loin derrière avec $0,37 \%$ et Pully, dont le contingent est formé d'une majorité de directeurs ou de «cadres», $0,2 \%$.

L'étude de la répartition de ces travailleurs est intéressante. Elle montre en effet clairement que la très grosse majorité des ouvriers «étrangers» travaillant à Renens se recrute dans la banlieue lausannoise, quoique le centre d'attraction soit, cette fois, situé dans la périphérie. ${ }^{15}$ Cette observation met en lumière le fait qu'entre la banlieue et la zone de voisinage se situe une limite importante. L'influence urbaine ne semble pas décroître régulièrement dans une zone concentrique. Elle décroît régulièrement jusqu'à une certaine limite, celle de la banlieue, puis il se produit une rupture de continuité. ${ }^{16}$ La banlieue apparaît, dans les mouvements de travailleurs, comme un monde semifermé, qui se distingue profondément de la zone de voisinage.

Cette constatation se fait beaucoup plus évidente si l'on groupe Lausanne et Renens (comme s'il s'agissait de la même ville) et que l'on additionne ainsi leurs forces d'attraction.

Les modifications sont surtout sensibles à l'ouest. De l'extérieur vers l'intérieur, les plus importantes sont celles qui affectent Gollion (4,5-5,7\%), ${ }^{17}$ Vufflens-la-Ville $(5,7-6,3 \%)$, Villars-Ste-Croix $(1,9-4,3 \%)$, Mex (2-4\%), Lonay $(7-7,9 \%)$, Echandens (9-9,75\%), Denges $(9,3-10,2 \%)$, Bussigny $(9,1-11 \%)$, Ecublens $(10-$ $14 \%)$, Crissier $(9,1-14,5 \%)$ et Chavannes $(12-18,9 \%)$. A l'exception de la région Echandens-Lonay-Denges, où l'influence perturbatrice de Morges se manifeste dans sa plus grande intensité, l'écart entre la banlieue (telle que nous l'avons définie dans le chapitre II) et la zone qui l'entoure extérieurement est plus nettement marqué que si l'on ne tient compte que de l'influence de Lausanne.

15 Seules quatre communes rurales envoient à Renens des contingents supérieurs à $1 \%$ de leur population: Gollion $(1.21 \%)$, Mex $(2 \%)$, Moiry $(1.33 \%)$ et Villars-Ste-Croix $(2.5 \%)$. Les trois dernières citées ont des populations inférieures à 200 personnes.

${ }^{16}$ M. R. Clozier fait une remarque du même ordre dans son « Essai sur la banlieue»: «La limite de la banlieue coïncide avec une chute brusque de la densité.»

17 Le premier coefficient est celui des travailleurs se rendant à Lausanne. Le second correspond au total des travailleurs occupés à Lausanne et à Renens. 


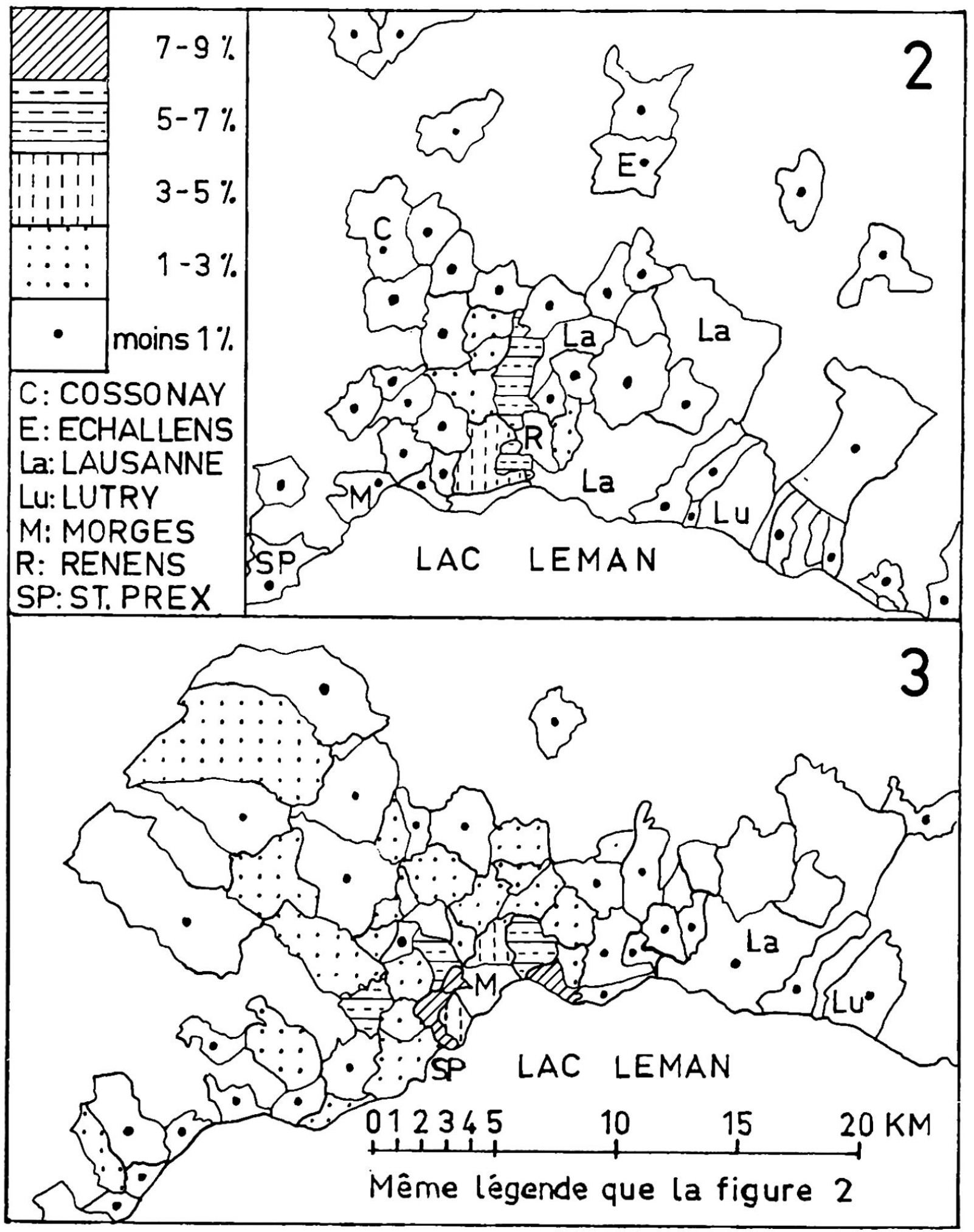

2. Provenance, en $\%$ de leur population de résidence, des personnes occupées à Renens. 3. Provenance, en $\%$ de leur population de résidence, des personnes occupées à Morges.

Nous ne pouvons pas ici nous étendre sur les causes de ce phénomène. Très brièvement dit, il semble que se soit la conséquence du fait que la banlieue est peuplée de personnes ayant reflué de la ville, et dont, par conséquent, tous les intérêts sont urbains, tandis que la zone de voisinage est encore psychologiquement rurale. La banlieue est un monde spécial de citadins disséminés. Or un noyau industriel, même très excentrique, fait appel en premier lieu à une main d'œuvre déjà «urbanisée» qui se trouve dans la banlieue et non pas dans la zone de voisinage. 
Nous pouvons clore ce chapitre en constatant qu'il apporte une confirmation de notre «première approximation»: la banlieue définie comme l'ensemble des communes dont plus du $9 \%$ de la population travaille à Lausanne. Mais cette confirmation n'est que partielle: dans la région de Lonay et d'Echandens les nouvelles informations ne suffisent pas à affermir nos hypothèses précédentes. C'est le moment de tirer le problème au clair, en déterminant l'influence de Morges.

\section{LA ZONE D'INFLUENCE DE MORGES}

Morges n'excerce sur les travailleurs qu'une attraction d'intensité assez médiocre: 490 personnes seulement travaillent dans cette petite ville et habitent dans d'autres communes.

Les mouvements normaux ne dépassent donc guère le $6 \%$ de la population et équilibrent avec peine les départs vers Lausanne (415) et Renens (29). Rappelons, à titre de comparaison, que Renens, commune typique de banlieue, attire 1265 travailleurs «étrangers», quoique sa population ne soit pas beaucoup plus importante (9600 personnes contre 8011).

La faible influence de la ville apparaît bien plus évidemment si l'on étudie la zone de recrutement de ces travailleurs, telle qu'elle figure sur notre carte no III.

Le nombre des communes qui envoient de la main d'œuvre est, comme à Renens et Lausanne, assez grand. Mais les contingents sont très faibles. Dans la plupart des cas, il ne s'agit que d'un ou deux ouvriers (on trouvera le détail de ces mouvements migratoires dans notre tableau de la page 89). 29 communes envoient un nombre de travailleurs représentant plus du $1 \%$ de leur population (et il s'agit de populations rurales, donc peu denses). ${ }^{18}$ De ces 29 communes, 6 seulement atteignent un «taux» d'émigrants alternants supérieur à $5 \%$ : Vufflens-le-Château $(5,12 \%)$, Villars-sous-Yens $(6,62 \%)$, Lonay $(6,98 \%)$, Lully $(7,68 \%)$, Préverenges $(7,87 \%)$ et Chigny $(8,33 \%)$. De ces six localités quatre comptent moins de 200 habitants, et envoient, au total, 40 de leurs habitants travailler au chef-lieu. On ne peut donc parler, dans ce cas, de banlieue caractérisée. Les deux communes qui restent, Lonay ( 945 habitants 6,98\%) et Préverenges (468 habitants 7,87\%) sont les seules dont le cas nous intéresse. Ces deux «rescapées» ne sont d'ailleurs pas dans une situation bien brillante: aucune n'atteint la cote de 9 à $10 \%$ que nous avons fixée comme limite de la banlieue. Leur densité de population est de 2,6 (Lonay) et 2,5 (Préverenges) habitants par ha., ce qui est faible. Ces chiffres sont caractéristiques de la zone de voisinage, ou de la grande banlieue.

En fait Morges n'a pas de banlieue. C'est une ville trop petite. Deux questions se posent toutefois: la ville fait-elle partie de la conurbation lausannoise? Si non, où se situe la limite entre les deux zones d'influences?

\section{Liste des communes dont plus de ro habitants sont occupés à Morges}

\begin{tabular}{lrrrr} 
& Population & \multicolumn{2}{c}{ Travailleurs } & $\%$ \\
& & H & F & \\
Aubonne & 1662 & 17 & & 1.00 \\
Echichens & 341 & 12 & 2 & 4.10 \\
Lausanne & & 56 & 17 & \\
Lonay & 945 & 55 & 12 & 6.98 \\
Lully & 170 & 10 & 3 & 7.68 \\
Préverenges & 468 & 32 & 5 & 7.87 \\
Renens & 9681 & 22 & 5 & \\
Saint-Prex & 1782 & 22 & 8 & 1.68 \\
Tolochenaz & 430 & 12 & 6 & 4.02 \\
Vufflens-Château & 195 & 10 & & 5.12
\end{tabular}

1810 communes seulement envoient plus de 10 personnes travailler à Morges. 
Il est sans doute tentant, dès l'abord, de faire entrer Morges dans la banlieue lausannoise, avec, bien entendu, toute la région qui sépare les deux villes. On obtiendrait ainsi une vaste agglomération de forme vaguement triangulaire, symétrique de part et d'autre d'un axe nord-sud, s'étendant sur plus de 20 kilomètres le long du lac. Or, la réalité est un peu différente, bien que l'aspect de la banlieue ouest puissent faire croire qu'il ne s'agit que d'une agglomération: de Morges à Lausanne on ne sort pas d'un paysage de moyenne banlieue, on ne retrouve nullepart un paysage purement rural.

Pourtant, quelques faits nous empêchent d'opérer cette simplification. Le plus frappant est sans doute qu'entre Lausanne et Morges, les échanges de travailleurs sont très limités: Morges n'envoie que 415 personnes à Lausanne (dont 59 écoliers et 44 apprentis) ce qui est peu pour une commune de 8000 habitants (Lutry, 3376 habitants, compte 465 émigrants alternants). Le courant inverse est bien plus faible encore, avec 67 personnes seulement. Et, dans ce cas, il existe une industrie locale, contrairement à ce qui se passe à Pully et Prilly, qui a besoin de main d'œuvre.

La comparaison avec Renens met bien en valeur la différence qui sépare Morges d'une ville de banlieue: des 1265 personnes que Renens attire, 598, près de la moitié, viennent de Lausanne. A peine plus du dixième de ceux que Morges attire viennent de la capitale. La distance qui sépare Lausanne de Morges et les difficultés de transport jouent-elles un rôle? Nous ne le pensons pas. Les moyens de transport ne créent pas toujours des courants, mais les facilitent quand ils sont établis. Les nouveaux quartiers attirent les lignes de bus ou de tram (ou de métro) plus souvent qu'elles ne se prolongent dans des zones inhabitées pour y créer des possibilités de résidence. Enfin une commune comme St-Sulpice, nettement tournée vers Lausanne est, à tout prendre, plus mal desservie (par les autobus) que Morges par le chemin de fer. Il serait donc faux de se fonder sur des considérations telles que l'éloignement ou le manque de transports suburbains pour donner aux 5,2\% de Morges une importance qu'ils n'ont pas et faire entrer la ville dans la conurbation.

La faiblesse des échanges de travailleurs entre Lausanne et Morges ne se manifeste pas seulement d'une ville à l'autre. Il est caractéristique de constater que celle-ci recrute sa main-d'œuvre dans la région sise à l'ouest plutôt que dans la banlieue lausannoise (pourtant très proche). La limite que nous avons précédemment fixée à celle-ci n'est pas loin de coïncider avec celle qui sépare, sur la carte no III, les communes dont le taux est inférieur à $1 \%$ des autres. Ensemble, les communes de Bussigny, Chavannes, Crissier, Ecublens et St-Sulpice n'envoient que 19 personnes travailler à Morges, ce qui fait une de plus que Yens, Villars-sous-Yens et Denens réunis! Cet équilibre apparent montre bien dans quelle mesure Morges échappe à la banlieue lausannoise et s'en détourne.

En fait, il semble que cette ville qui, à la différence de Renens, Pully, ou d'autres communes suburbaines, a une très longue histoire urbaine derrière elle, et qui joue un rôle de capitale régionale depuis fort longtemps, continue à remplir cette fonction. L'industrie n'a pas amené de bouleversement et la ville reste tournée vers son pays.

Puisqu'il n'y a pas de conurbation, il faut chercher à définir les zones d'influences respectives de Morges et Lausanne. C'est une tâche assez simple. Il suffira de trouver la zone où les deux attractions se manifestent avec une intensité égale.

Nous avons vu que la force d'attraction de Morges diminue très rapidement en direction de Lausanne: Bussigny, E.cublens et St-Sulpice font indiscutablement partie de l'ensemble lausannois. A Denges et à Echandens, l'influence morgienne se fait un peu plus forte. Elle n'atteint cependant qu'une petite proportion de celle de la capitale: un sixième environ. A Préverenges, la lutte devient plus égale: Lausanne reçoit quotidiennement le 6,8\% de la population, Morges le 7.87\%. L'équilibre est renversé. Encore faut-il ajouter que le contingent lausannois comprend 9 écoliers et apprentis, tandis 
que celui de Morges est constitué de travailleurs seuls. Nous n'avons pas recensé les écoliers de cette commune se rendant à Morges. Il y en a sans doute.

L'important est qu'ici l'influence de Lausanne est battue en brèche par celle de la ville voisine. Préverenges échappe à la banlieue lausannoise. Le cas de Lonay est semblable quoiqu'un peu moins net: 66 personnes (dont 52 travailleurs) vont à Lausanne, 67 travailleurs vont à Morges. Le contingent d'écoliers ferait pencher la balance de façon plus marquée vers Morges.

Nous limitons l'étude de la zone d'influence de Morges à ces quelques communes, situées dans la zone où la concurrence entre les deux villes se fait sentir. Elle nous a permis de constater que le taux de $9 \%$ d'émigrants alternants que nous avons choisi pour définir la banlieue correspond vraiment à une réalité.

A l'issue de ce chapitre, la banlieue semble définie, ou, tout au moins, la méthode que nous avons choisie est au bout de ses possibilités. En fait, la question de la limite nord de la banlieue reste vague. Il nous est impossible, pour les raisons que nous avons évoquées plus haut, de la définir par l'étude des migrations alternantes. Nous allons tout de même tenter, dans le chapitre suivant, d'en préciser un peu l'extension, en nous fondant sur l'observation directe et sur l'étude de la carte.

\section{LA LIMITE NORD}

Le territoire de la Commune de Lausanne revêt une forme assez curieuse, avec ses deux parties presque carrées, reliées par un «corridor» étroit et entre lesquelles vient s'insérer la Commune d'Epalinges. Les conséquences sont que ce territoire, complètement urbanisé dans la région basse, s'étend, au nord d'Epalinges, sur une région beaucoup plus rurale, voire forestière, et qu'on est dans l'impossibilité de distinguer par l'étude des mouvements de travailleurs où s'arrête la ville et où commence la banlieue.

Administrativement, il n'existe aucune différence entre les diverses parties de ce territoire. Ainsi, par exemple, la population de Lausanne indiquée officiellement compte aussi les habitants de la région haute, quoiqu'ils n'habitent évidemment pas la ville.

Où commence la banlieue? Il ne nous reste, pour la préciser, que le recours à une carte à grande échelle, la carte nationale au 1:50 000 par exemple. On y distingue un noyau central où les constructions sont contiguës, assez restreint, puis les espaces non bâtis apparaissent et gagnent progressivement en importance. La transformation est presque imperceptible aussi est-il assez difficile de trancher.

La limite supérieure de la ville semble se situer vers 550 mètres d'altitude. Au-delà apparaissent les vastes espaces libres de la banlieue: stades, station fédérale d'essais agricoles.

Une seconde limite apparaît vers 600 mètres, à la hauteur de la Sallaz. En-dessous les bâtiments se répartissent sur tout le territoire de la commune, tandis qu'au-dessus leur implantation semble être influencée par les voies de communications et les noyaux préexistants. La densité de peuplement devient plus faible. Les maisons sont plantées dans des espaces non bâtis de plus en plus vastes voués à l'exploitation agricole.

Où s'arrête l'influence urbaine, la banlieue? En interprétant aveuglément notre carte no I, nous serions amené à dire que la frontière communale marque la limite de la banlieue. Une telle déclaration serait risquée, vu le manque total d'éléments d'estimation. Elle ne serait, par hasard, pas trop inexacte!

Jusqu'à la lisière sud de la forêt, dans la région du Chalet-à-Gobet et du nord d'Epalinges, le paysage de banlieue se maintient. Banlieue extérieure, sans doute, dont le peuplement est très dispersé. Mais les maisons suburbaines, les villas, dont l'unique fonction est la résidence de gens travaillant ailleurs, sont encore plus nombreuses que les fermes. Enfin, la présence du terrain de golf, quoiqu'elle ne se marque guère dans le paysage, est un témoin de l'influence et de la proximité de la ville. La forêt elle-même 
est altérée par le voisinage de l'agglomération. De nombreuses bonnes routes, de nombreux chemins et sentiers balisés la traversent en tous sens. Nous sommes en pleine zone de villégiature, envahie de promeneurs à la belle saison.

Est-il utile de chercher une limite précise? Peu, sans doute. Il est indéniable que la limite de la commune ne marque que très approximativement la fin de la banlieue, qui s'arrête probablement avant. On pourrait proposer la lisière de la grande forêt qui va de Cugy au Chalet-à-Gobet, en restant peut-être un peu en deçà de la réalité. Mais cette limite, pour être un peu plus exacte, est moins connue, moins facile à repérer. C'est pourquoi nous pensons préférable d'admettre ici que la frontière communale circonscrit la zone suburbaine au nord de la ville.

D'après notre carte no I et la limite de $9 \%$ que nous avons fixée, il faudrait considérer Montpreveyres comme une commune de banlieue. Mais la réalité s'oppose sur ce point à nos statistiques. Ce village, dont $9 \%$ de la population exerce son activité à Lausanne, ne compte plus que 208 habitants (décembre 1958). Ce chiffre est bien inférieur à celui de 1950 (243 habitants). Le village accusant en huit ans une perte de population de plus de $15 \%$ ! Et ce n'est pas un phénomène passager, puisque la population de 1910 était de 280 personnes, et celle de 1860 de 270 personnes. ${ }^{18}$ C'est la preuve que la banlieue, qui se signale par un brusque accroissement de la population, n'a pas encore atteint ces régions. Enfin la densité de population, proche de 0,5 habitants par ha. pour l'ensemble du territoire communal, est inférieure à l'habitant par ha. si l'on défalque la surface boisée; elle est nettement en-dessous de la plus basse densité de la banlieue (celle de Romanel, qui atteint 1,2 habitants par ha. pour l'ensemble de la commune).

Enfin, si l'on examine la composition du contingent de travailleurs de Montpreveyres exerçant leur activité à Lausanne, on constate que, sur un total de 17 personnes, il y a 8 ouvriers (dont une femme seulement) et 5 manœuvres. L'importance de cette proportion montre que nous sommes, à Montpreveyres, dans une commune où la ville attire la population rurale (apte à exécuter des travaux de force, mais peu spécialisée) et non, comme c'est le cas dans la banlieue, dans une zone où reflue l'excédent de population urbaine.

Il faut ainsi se rendre à cette évidence que, même le long des chemins de fer de Moudon, la banlieue ne dépasse pas les limites de la Commune de Lausanne.

\section{LA BANLIEUE MARAICHËRE}

Les maraîchers ne peuvent pas être comptés dans les travailleurs participant aux migrations alternantes. Leurs déplacements, cependant, pour être moins fréquents (2 ou 3 par semaine) n'en ont pas moins une grande importance: ce sont aussi des gens qui gagnent la plus grande partie de leurs revenus sur territoire urbain. C'est pourquoi nous nous occupons d'eux dans ce travail.

La culture maraîchère est ce qui reste d'une des plus anciennes fonctions de la banlieue: le ravitaillement de la ville en produits frais. Son extension fut donc limitée longtemps par la lenteur des moyens de transport. Bien que cela ne soit plus le cas aujourd'hui, elle se maintient dans la zone suburbaine.

Il s'est révélé impossible de faire le compte exact des maraîchers prenant part aux marchés de la ville. La statistique tenue par la «Caisse de police» est basée sur le nombre «d'emplacements» loués par la ville. Les emplacements non contigus loués par une même personne apparaissent donc deux fois dans le fichier, sans que l'on puisse s'en

19 « Dictionnaire historique, géographique et statistique du Canton de Vaud.» 


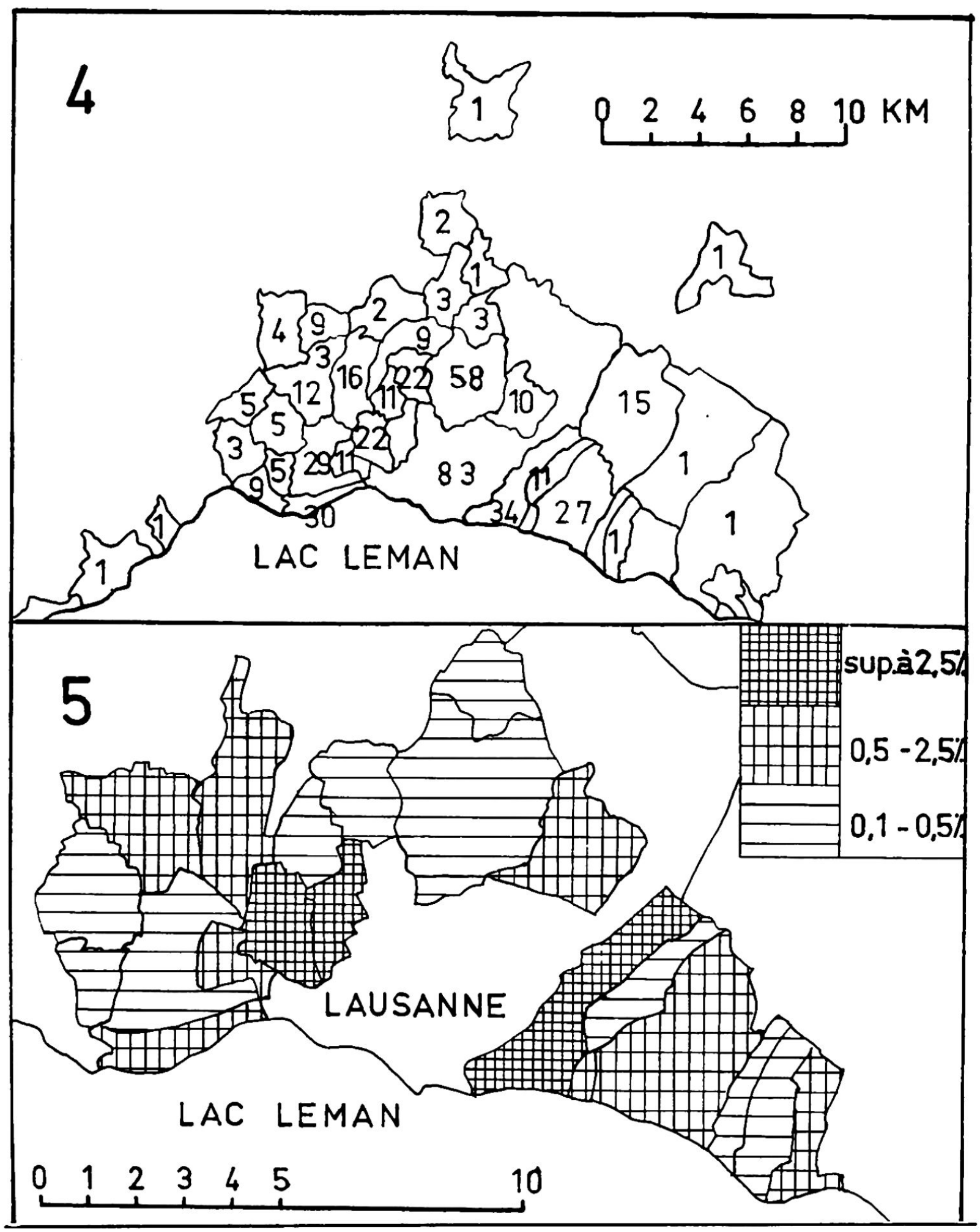

4. Communes de résidences des maraîchers participant aux marchés lausannois. (Les chiffres indiquent le nombre d'emplacements loués par les maraichers de la commune considérée aux marchés de Lausanne). 5. Densité des personnes soumises ì des mouvements alternants quotidiens vers Lausanne. (Les chiffres indiquent le nombre d'a émigrants alternants p par ha du territoire communal. Les surfaces forestières ont été déduites des superficies communales).

rendre compte, car le classement est effectué par ordre alphabétique et par rue, ce qui fait que les fiches d'une même personne ne sont pas groupées. Aussi les chiffres que nous donnons sont-ils inexacts: ils n'indiquent pas le nombre de maraîchers d'une commune, mais le nombre de «places» louées par les maraîchers de cette commune. Faute de mieux, nous avons dû nous contenter de cette solution, qui sans doute reflète la situa- 
tion sans trop la déformer, car le nombre de places que loue un maraîcher est, la plupart du temps, en rapport direct avec l'importance de son exploitation.

Parmi les maraîchers qui participent aux marchés de Lausanne, une grande partie habite la commune même (92). Les communes qui envoient les plus forts contingents sont, dans l'ordre: Le Mont (58), Prilly (38), Pully (34), St-Sulpice (30), Ecublens (29), Lutry (27), Romanel (22), Renens (22), Crissier (16), Savigny (15), Bussigny (12), Chavannes (11) et Epalinges (10). Parmi ces communes, une seule n'est pas comprise dans la banlieue telle que nous l'avons définie: Savigny. Toutes les autres confirment ce que nous avons observé plus haut: La banlieue est un milieu très différent de la zone qui l'entoure à l'extérieur. La culture maraîchère est une activité spécifique. de la banlieue 20 exigeant une main d'œuvre assez nombreuse, qui se défend mieux contre l'envahissement par les maisons qu'elle ne s'implante dans un terroir rural. Un exemple éminent est celui de Pully, qui se classe au troisième rang avec 34 maraîchers, alors que ces exploitations se trouvent cernées de toutes parts par les immeubles et que le mètre carré de terrain atteint, par le jeu de la spéculation, des prix extrêmement hauts. Et si Pully compte 34 maraîchers, Lutry où pourtant les terrains non bâtis sont bien plus abondants n'en compte que 27. (La chute brusque du nombre de marấchers ne permet pas de pousser plus loin cette comparaison, car à l'ouest de Lutry apparaît un phénomène nouveau: la présence de la vigne sur un coteau particulièrement favorable.)

Si l'on observe maintenant la région supérieure de la banlieue, dont le climat est beaucoup moins favorable, on fait la même constatation: les maraîchers du Mont louent 58 places, ceux de Savigny 15 et ceux de Forel 1. Or la question des transports ne joue ici qu'un rôle secondaire. Le trolleybus du Mont facilite l'installation de banlieusards, mais il n'est pas utilisé pour transporter les corbeilles au marché! Ici c'est la camionnette qui domine et elle efface les différences locales.

Ceci dit, il paraîtra moins étonnant que la banlieue maraîchère recouvre, sur notre carte, la banlieue tout court! Toutes les communes dont les maraîchers louent 5 places ou plus dans les marchés lausannois sont des communes dont plus du $9 \%$ de la population est occupée en ville, à l'exception de Savigny, de Mex, de Préverenges, et de Bremblens sur Morges. Encore peut-on faire intervenir, pour expliquer le fait que ces deux dernières communes fassent partie du groupe, la proximité de Morges, qui, ajoutant les débouchés de son marché à ceux de Lausanne, crée une situation particulièrement favorable.

\section{LES DIVERSES ZONES DE LA BANLIEUE}

L'étude des mouvements journaliers de travailleurs nous a permis de fixer les limites extérieures de la banlieue lausannoise. Elle ne nous a guère mis en mesure, par contre, de distinguer les diverses zones de la banlieue. Cela tient au fait que nous avons constamment utilisé des chiffres relatifs (à la population totale). Cette manière de faire ne tient aucun compte de l'importance réelle des localités dont nous avons parlé: elle met, comme nous l'avons déjà relevé, sur le même pied des communes aussi différentes que Renens et Le. Mont.

Or, l'aspect d'une région de la banlieue est déterminé, dans une large mesure, par sa densité de population, qui dépend de la densité des.constructions, de l'étendue des terrains non bâtis et qui provoque la plus ou moins grande congestion des moyens de transports.

Il est impossible, dans un travail de cette étendue, de faire une analyse des densités de population des communes suburbaines, étude réputée longue et délicate, qui exigerait

20 A l'exception du cas, bien entendu, où elle se développe sur une grande échelle pour devenir une culture spéculative. 
une tout autre organisation de ce travail. Mais nous pouvons remédier partiellement à cette lacune en calculant, dans chaque commune, le nombre d'émigrants alternants par ha. Nous obtiendrons ainsi une carte des mouvements de travailleurs qui tiendra compte, indirectement, des densités (carte V).

Le défaut d'un tel coefficient est d'être, peut-être, exagérément théorique. On a en effet de la peine à se représenter concrètement ce qu'il signifie. Mais ce défaut est compensé par certaines qualités non négligeables. Ainsi il a l'avantage sur le calcul ordinaire de la densité de ne tenir compte absolument que de l'élément suburbain. Une commune exclusivement rurale, même prospère et très peuplée, aurait l'indice zéro. C'est pourquoi cette manière de faire est d'une application moins délicate que celle qui se fonde sur les densités totales de population.

Ces indices seront toujours assez faibles. Il n'est peut-être pas inutile d'insister sur ce point pour éviter des erreurs d'interprétation. A cet effet, il faut signaler que le rapport des travailleurs occupés en ville à la surface communale est, à Lausanne même, de 22,4 seulement (chiffre calculé sur la base du recensement de 1950). La commune suburbaine qui atteint l'indice le plus élevé est Prilly, avec 7,1. Un indice de 1 doit être considéré comme moyen (il correspond en effet, puisqu'il s'agit de communes où 10 à $20 \%$ de la population travaille en ville, à une densité totale de 5 à 10 habitants par ha.).

Enfin, signalons que tous ces indices ont été calculés par rapport à la surface habitable des communes, c'est-à-dire à la surface non boisée (les forêts vaudoises, classées forêts protectrices, sont des îlots d'une stabilité à toute épreuve devant lesquels l'expansion urbaine doit céder).

Les indices des communes de la banlieue se répartissent en trois groupes: ceux qui sont supérieurs à 2,5 , ceux qui sont compris entre 0,5 et 2,5 , et ceux qui sont inférieurs à 0,5. Le premier groupe comprend les communes de Prilly $(7,1)$, Pully $(5,58)$, Renens (4,9). Le second: Paudex (2,31), Chavannes (1,2), St-Sulpice $(1,14)$, Crissier $(0,67)$, Cully $(0,56)$, Epalinges $(0,54)$, Lutry $(0,5)$ et Bussigny $(0,5)$. Le troisième est constitué par les communes de Belmont et Ecublens $(0,44)$, Villette $(0,43)$, Grandvaux (0,33), Le Mont (0,29), Jouxtens-Mézery (0,28), Cugy $(0,21)$, Denges $(0,2)$, Romanel $(0,17)$ et Echandens $(0,13)$.

Ces trois groupes statistiques correspondent évidemment à trois zones d'aspect bien différent. Le premier réunit les communes de la banlieue immédiate: Prilly, Pully et Renens.

Le deuxième est le groupe de la proche banlieue, zone où l'influence urbaine se fait encore sentir très fortement, mais où la densité devient plus faible, où les espaces non bâtis l'emportent nettement sur les constructions. L'exploitation agricole se maintient, mais perd rapidement du terrain. Elle est bientôt limitée aux régions les moins favorisées par les moyens de transport, ou aux régions où des conditions spéciales permettent le maintien d'une culture particulièrement rémunératrice. C'est la zone où refluent les industries les plus encombrantes, celle où l'on trouve le plus grand nombre de «dépôts». (Dépôts des entrepreneurs de travaux publics, des compagnies pétrolières, etc.).

Quant à la troisième zone, dont la densité est la plus faible, elle constitue ce qué l'on appelle souvent la «moyenne banlieue»21. (Nous préférons pour notre compte le terme de «banlieue périphérique» ou de «banlieue extérieure», parce que celui de «moyenne banlieue» suppose celui de «grande banlieue», et que la «grande banlieue» n'est, en fait, plus du tout la banlieue.) C'est la région «qui n'appartient plus du tout à la ville, mais qui en dépend directement» ${ }^{22}$. L'insolite est, dans ce paysage, l'élément suburbain et non plus l'élément rural. 
Ces trois zones semblent se répartir assez curieusement sur notre carte. Ainsi dans la région de Bussigny et Crissier, la proche banlieue paraît en contact direct avec la campagne. Ecublens, de son côté, peut paraître désavantagé. Il ne se distingue en effet plus de Denges ou d'Echandens... La cause de ces anomalies apparentes est, une fois de plus, que nous prenons la commune pour base. Les indices sont la «moyenne» des communes. Or certaines d'entre elles présentent diverses parties très différentes. C'est le cas notamment à Ecublens et Crissier qui touchent par une extrémité à l'agglomération de Renens-Gare, tandis que l'autre est en plein monde rural. Il n'est guère possible dans un travail comme celui-ci d'entrer dans plus de détail. Aussi signalons-nous la lacune sans pouvoir $y$ porter remède.

\section{LA ZONE D'INFLUENCE}

Ce travail n'avait pour but que de déterminer la limite de la banlieue lausannoise. Ce but semble atteint, mais il est tentant d'étudier, à l'aide des statistiques que nous possédons, la zone qui se situe en dehors de cette limite.

Nous disposons pour cette esquisse d'une base semblable à celle que nous avons utilisée jusqu'ici. En effet, d'après M. M. SorRe, l'influence urbaine s'étend à toutes les localités dont $10 \%$ des travailleurs sont occupés en ville (voir p. 81 ).

Une difficulté se présente immédiatement: il n'existe pas de statistique annuelle des personnes exerçant une activité professionnelle dans chaque commune. Nous la tournerons en extrapolant à partir des données du recensement fédéral de 1950. En calculant, dans les sept principales villes vaudoises (Lausanne, Vevey, Yverdon, Montreux-Châtelard, Pully, Morges, Renens et Payerne) la proportion que représentent les travailleurs par rapport à la population totale, nous obtenons respectivement les valeurs suivantes: $49,50,47,45,40,42,41$ et $42 \%$. Il est inutile de calculer une moyenne précise, vu qu'il s'agit d'extrapoler. Admettons la valeur de $45 \%$.

Si les travailleurs représentent le $45 \%$ de la population, le dixième du nombre des travailleurs représente le $4,5 \%$ de la population totale. Cette valeur va nous permettre de repérer, sur notre carte no 1 , la zone où s'étendent les «influences urbaines».

Cette zone est naturellement beaucoup plus vaste que la banlieue. Elle s'étend manifestement le long des voies de communications: chemin de fer de Vallorbe et de Berne. Tramway de Moudon, et région desservie par le Lausanne-Echallens-Bercher.

Comment nommer cette zone? Elle est bien plus réduite que la zone d'influence. Est-ce la grande banlieue? Nous avons dit plus haut le peu de bien que nous pensions de ce terme, dont le sens est mal précisé. (Ne le confond-on pas trop souvent avec zone d'influence, notamment quand on dit, par exemple, que toute la France est la grande banlieue de Paris?) Nous préférons le remplacer par celui de «zone de voisinage» que l'on a défini de la manière suivante (qui semble parfaitement convenir au cas de Lausanne) : «zone où les gros bourgs peuvent avoir une certaine autonomie, où la population s'emplace dans une industrie sur place, où la ville ne joue son rôle que pour ses grandes fonctions de capitale ou son activité commerciale. Elle apparaît aussi comme la zone de week-end, des promenades familiales; plus que les zones précédentes (banlieue) elle présente des paysages ruraux ou de montagne». ${ }^{23}$

Quant à la zone d'influence proprement dite, il est impossible de la définir sur la base des renseignements que nous possédons. Elle peut en effet dépasser largement la zone de recrutement de la main d'œuvre et s'étendre jusqu'au moment où elle se heurte à la zone d'influence d'une autre ville de même rang. p. 581.

${ }^{23}$ H. Vivıan: * La zone d'influence de Grenoble». Revue de Géographie alpine. 1959. Fasc. IV, 


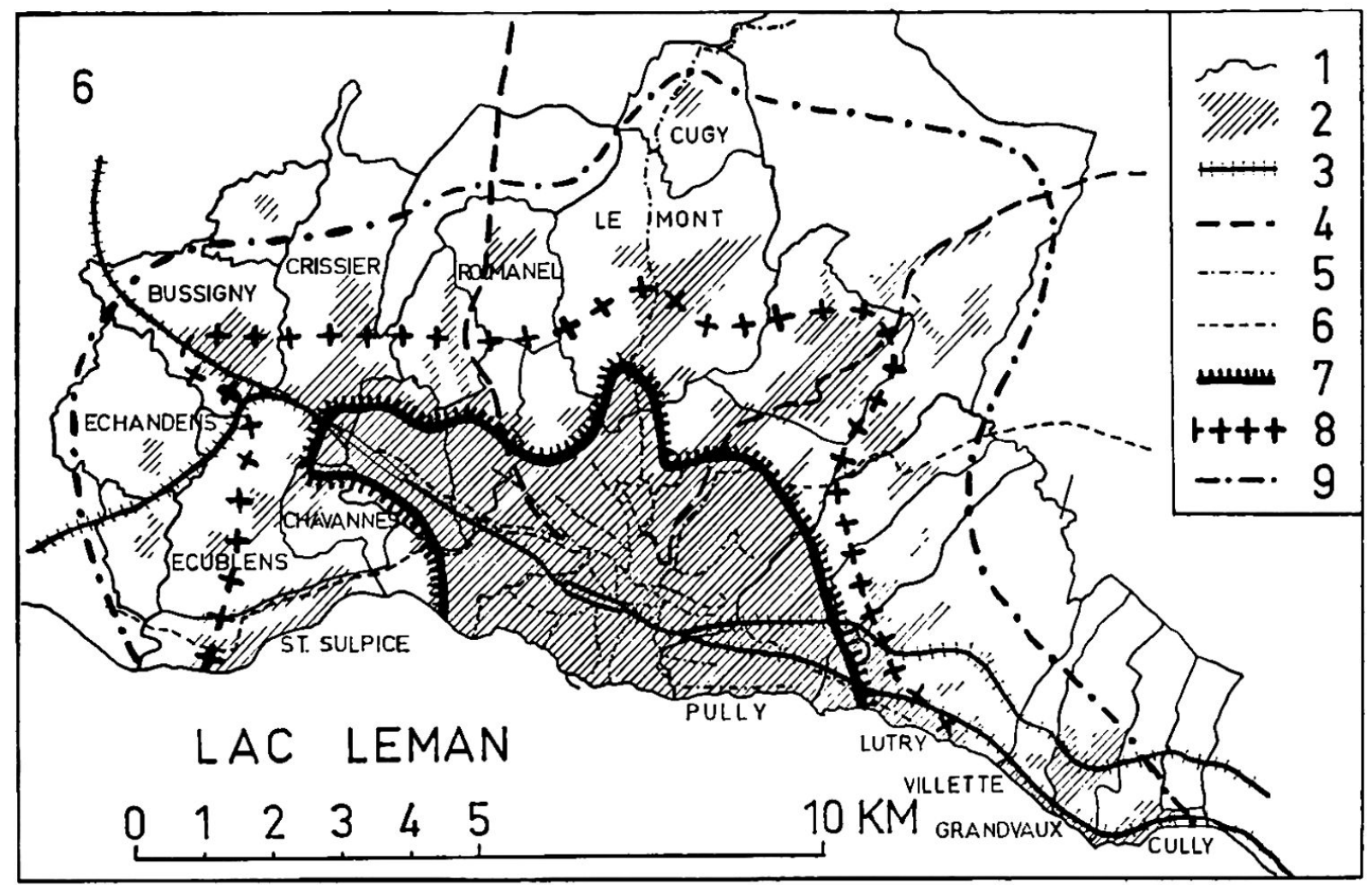

6. Essai de représentation de l'agglomération lausannoise. $1=$ Limites communales. $2=$ Surface bâtie. $3=$ Chemins de fer (CFF-SBB) $4=$ Chemins de fer à voie étroite. $5=$ Transports urbains (trolleybus et tramways). $6=$ Autobus suburbains. $7=$ Limite de la banlieue immédiate. $8=$ Limite de la proche banlieue. $9=$ Limite de la banlieue extérieure.

\section{CONCLUSION}

Nous avons tenté, au cours de ce travail, de préciser les limites de la banlieue lausannoise, en nous fondant presque exclusivement sur l'étude des mouvements de travailleurs. La complexité de cette entreprise se reflète dans le grand nombre de chapitres qui nous ont parus nécessaires. Il en résulte un certain manque d'unité auquel nous essaierons de remédier ici.

D'autre part, nous avons signalé plusieurs fois déjà que la nécessité de travailler en considérant chaque commune comme un tout ne va pas sans entraîner un certain nombre d'erreurs, dues au fait que la surface réellement occupée par la banlieue ne recouvre jamais le territoire communal en entier. Il est malheureusement impossible, dans un travail comme celui-ci, d'aborder chaque cas individuellement et d'examiner la structure de toutes les communes.

Nous nous contenterons donc de dresser une dernière carte, à plus grande échelle, de l'agglomération telle que nous l'avons définie. Sur cette carte, nous indiquons quelles sont les zones construites (sans tenir compte de la densité des constructions). Nous avonis été obligé de procéder de manière approximative, en marquant, dans les communes de la proche banlieue et de la banlieue extérieure, le périmètre des groupes de maisons, même quand celles-ci sont assez disséminées, et sans pouvoir distinguer les maisons de résidence suburbaine des fermes de caractère purement rural, ou des locaux industriels.

Nous avons également reporté sur cette carte le réseau des moyens de transport, et nous y avons tracé, approximativement, les limites des diverses zones de la banlieue (carte VI).

Nous espérons ainsi à la fois résumer et concrétiser ce que nous avons dit des limites de la banlieue, et en donner une image moins schématique que celles où les territoires communaux apparaissent comme des unités indifférenciées. 
Notre première intention était d'étudier, dans ce mémoire, la banlieue lausannoise. Il fallut bien se rendre à cette évidence qu'un tel sujet, beaucoup trop vaste, ne pouvait être traité ici; nous nous sommes contenté d'étudier l'un des phénomènes de la banlieue, les migrations alternantes, et nous avons obtenu certains résultats, qui souffrent de la nécessité dans laquelle nous nous sommes vu de ne nous attacher qu'à un des aspects de la question: ce travail se ressent de l'application exclusive d'une méthode. Ses conclusions doivent être considérées comme des hypothèses, qui devraient pouvoir être confirmées par d'autres études, spécialement par celle des densités de population.

Aussi ce mémoire laisse-t-il, sur bien des points, espérer d'autres développements. Une carte comme celle de la page, où sont représentés les mouvements de travailleurs dirigés vers Lausanne est d'un indiscutable intérêt pour la connaissance des régions rurales du Canton de Vaud. A quoi tiennent les différences assez marquées que l'on observe, quant au nombre de leurs habitants occupés en ville, entre les diverses régions? Y a-t-il un rapport entre le nombre «d'émigrants alternants» et les systèmes fonciers ou les modes d'exploitation agraires? On pourrait multiplier ces questions à l'infini. Elles ne pouvaient être abordées dans ce travail. Nous espérons qu'elles le seront un jour. ${ }^{24}$

\section{DAS LAUSANNER STADTGEBIET IM LICHTE DER PENDELWANDERUNG}

Die Entwicklung der Lausanner Agglomeration hat die Landschaft der benachbarten Gemeinden tiefgreifend verändert und die Stadt zieht täglich eine erhebliche $Z$ ahl von auswärtigen Arbeitern an. Es war daher gegeben, bei einem Versuch das Weichbild der Stadt zu bestimmen, auf einer Untersuchung der Pendelwanderung zu fußen. Jeden Tag verlassen etwa 11000 Personen 229 waadtländische Gemeinden, um sich in den Hauptort zu begeben, der sie durch verschiedene Industrien oder Schulen anzieht. Mehr als ihre absolute Zahl ist es der Prozentsatz der Pendler, welcher die Intensität des städtischen Einflusses zu ermessen erlaubt, und Karte 1 zeigt klar die Abnahme dieser Einwirkung mit zunehmenden Entfernungen der $\mathrm{Ge}-$ meinden von der Stadt. Indessen markiert eine durch die Gemeinden, von welchen mehr als $9 \%$ der Bevölkerung in Lausanne beschäftigt sind und die übrigen bestimmte deutliche Unterbrechung die Grenze des Weichbildes.

Es waren auch die gegen zwei sekundäre Zentren gerichteten Arbeiterwanderungen: nach Renens (das Teil des städtischen Weichbildes ist) und Morges zu studieren. Dabei zeigte sich, da $\beta$ die große Mehrheit der Arbeiter aus Renens den übrigen Gemeinden des Vorortsgebietes entstammen und man von einer Conurbation (Karte 2) sprechen kann. Im Gegensatz dazu rekrutiert Morges seine auswärtigen Arbeiter in einer andern, mehr im Westen gelegenen Region (Karte 3). Diese ersten Untersuchungsergebnisse bestätigen das Studium der «Gemüsezone», indem die überwiegende Mehrheit der die Lausanner Märkte beliefernden Gärtner aus dem Vorortsraum stammen. Wenn man schlieBlich die Volksdichte mit dem Prozentsatz der in Lausanne beschäftigten Vorortsbewohner kombiniert, sondern sich undiskutierbar die folgenden eigentlichen Vorortsgemeinden von den übrigen: Prilly, Pully, Renens, Paudex, Chavannes, StSulpice, Crissier, Bussigny, Epalinges, Lutry, Grandvaux, Villette, Belmont, Le Mont, Cugy, Jouxtens-Mézery, Romanel, Ecublens, Denges und Echandens.

Der städtische Einflu $\beta$, der rapid in der Richtung des Lavaux abnimmt, verlängert sich ebenso deutlich nach Westen, wo kein Unterbruch der Kontinuität zwischen der Lausanner Agglomeration und den ökonomisch von Morges abhängigen Gemeinden mehr besteht.

24 Cette étude fut présentée à la Faculté des Lettres de l'Université de Lausanne pour l'obtention de la licence ès lettres. Elle fut réalisée dans le cadre de l'«Institut de Géographie de l'Université de Lausanne», et nous tenons à exprimer en premier lieu notre gratitude à son directeur, Monsieur le Professeur Henri Onde. Nous avons également contracté une grosse dette de reconnaissance envers Monsieur Georges-AnDRÉ Chevallaz, Syndic de Lausanne, sans l'appui duquel ce travail n'aurait pas pu être réalisé, et la Municipalité de Lausanne qui rendit possible sa publication par une généreuse subvention. Il convient de citer et de remercier ici MM. ARThUR Bürki, Syndic de Renens et Bonardel, boursier de la Commune de Morges; M. LOUIS VIRET, Chef du service communal des impôts de Lausanne et, de façon générale, les employés de la Commission communale des impôts, des Greffes municipaux de Renens et de Morges, du «Service cantonal de l'enseignement supérieur» du «Service cantonal de la formation professionnelle», et de la «Caisse de police» de Lausanne, qui répondirent avec la plus grande obligeance à nos questions et mirent aimablement les renseignements qu'ils détenaient à notre disposition. 


\title{
B I B LIOG RAPH IE
}

\section{Statistiques}

*Annuaire officiel » du Canton de Vaud. Ed. 1959-1960. Chancellerie d'Etat, Lausanne. «Annuaire statistique de l'Union des villes suisses. Ed. 1956. Tableau no. 6. « Dictionnaire historique, géographique et statistique du Canton de Vaud». Lausanne. Ed. F. Rouge. 1914.

\section{Sources manuscrites}

Fichier des « Hors Lausanne» de la Commission d'Impôt de la Commune de Lausanne. Listes d'effectifs des entreprises de Renens, consultées au Greffe municipal de Renens. Listes d'effectifs des entreprises de Morges, consultées au Greffe municipal de Morges. Fichier des maraichers louant des emplacements aux marchés de Lausanne. Consulté à la * Caisse de police*.

\section{Livres et articles}

ED. Berthaux: "Des études sur le phénomène de banlieue ». Revue Géographie de Lyon. 1950. III p. 242. P. Bonnoure: « Etude sur les éléments de l'organisme urbain. I. La banlieue ". Revue Géographie de Lyon. 1950. H. Carol: * Die Wirtschaftlandschaft und ihre Kartographische Darstellung . Geographica Helvetica. 1946. P. 246 et suiv. G. Chabor: "Les Villes ". Coll. A. Colin. Paris 1948. A. Chatelain: a Les notions démogéographiques des zones urbaines: de la cité à la banlieue •. Revue de Géographie de Lyon. 1946. A. Chatelain: Les faces démogéographiques d'expansion urbaine ». Revue de Géographie de Lyon. 1950. No. 3 p. 215-218. A. Chatelain: “ Les banlieues et les transports automobiles». Revue de Géographie de Lyon. 1951. R. CloziER: « La Gare du Nord . Thèse. Paris 1940. R. Clozier: «Essai sur la banlieue La Pensée », nouvelle série, no 4. juillet-septembre, p. 48-57. F. НАСКн : Der Personen-Nahverkehr ". Thèse. Winterthur 1955. P. Lavedan: « Géographie des villes ». Coll. Géographie humaine. NRF. Paris 1936. W. SCHÄrER: * Die suburbane Zone von Zürich». Thèse. Zurich 1956. M. Sorre: *Les fondements de la géographie urbaine». Tome III. H. VIVIAN: «La zone d'influence régionale de Grenoble». Revue de Géographie alpine. Grenoble 1959. Fasc. IV p. 539 à 585.

\section{DIE LANDSCHAFT DER TALAYOTS}

\section{Friedrich SPRINGORUM}

\author{
Mit Farbtafel
}

Nichts ist so schwer zu verteidigen und so leicht $\mathrm{zu}$ überfallen wie die durch unzählige Buchten und Vorgebirge noch verlängerte und unübersichtlich gewordene Küste einer Insel. Auf Mallorca haben die Römer das schon gewußt, ebenso später die Araber und die mallorquinischen Könige. So ist rund um die Insel, auf allen Berggipfeln, eine Kette von untereinander durch Sicht verbundenen Wachtürmen entstanden, die durch Feuerzeichen die Bevölkerung vor dem Herannahen der Piraten warnen konnten. Viele von diesen Atalayas sind zerfallen, viele auf steilem Fels schwer zugänglich; zu den schönsten zählt der Atalaya des Animes, der Turm der Seelen zwischen Banyalbufar und Estellenchs. Die Fernsicht von hier erfaßt ein großes Stück der Nordküste. Selten übersieht man eine so unendlich weite Meeresfläche; an ihrer absoluten Leere spürt man, was das ist: das Meer.

Von den Talayos auf Mallorca weiß man nicht viel mehr, als daß sie existierten. Spüren wir jedoch Größe und Dichtigkeit ihrer Existenz, so begnügen wir uns nicht mit dieser geringen Kenntnis, sondern möchten mehr erfahren. Ihrem Alter nach gehören die Talayots in die zweite Bronzezeit Mallorcas. Die einen Archäologen sehen in ihnen Verteidigungsbauten eines unbekannten und untergegangenen Volkes, andere stark befestigte Fürstenburgen, wieder andere Grabstätten oder auch alles zusammen. Der Name Talayot sagt nichts aus, er stammt von dem spanischen Wort Atalaya, das Wachtturm bedeutet. In Sardinien, wo es ähnliche, meist größere und besser erhaltene Bauten gibt, werden diese Nuraghe genannt. Die meisten Talayots Mallorcas sind zerstört, verschüttet, abgetragen und überwachsen. Nur wenige, notdürftig ausgegraben, bieten von außen gesehen, ein einigermaßen vollständiges Bild. 